\title{
National Vulnerability Assessment System: Part 2 Model Testing Fengshan Song
}

\author{
School of Control and Computer Engineering, North China Electric Power University, Baoding,
} China.

\begin{abstract}
In order to further determine the impact of climate change on national vulnerabilities, we choose Yemen as the study object. On the one hand, we select some countries that have been in an extremely fragile state for a long time as a reference standard. With method of Grey Relational Analysis, we calculate rational grade of each metric by use of related data collected from the World Bank. On the other hand, we use regression analysis to find the regression equation between climate change and the four indicators. The results show that the lack of precipitation and high temperature in Yemen has largely affected the second-class indicators, thereby increasing the country's vulnerability. in the case of Yemen, we have put forward some interventions and analyzed how these measures can effectively prevent a country from becoming a vulnerable country. Finally, we test the sensitivity of our model, conclude the strengths and weakness. And we discuss the applicability of the model to states of different sizes. We conclude that the model is applicable in smaller states and not in larger states, and improve the model.
\end{abstract}

Keywords: Climate change, NVAS, grey relational analysis, regression analysis.

\section{Introduction}

The fragile state has increased the pressure of the national population in the face of climate shock such as natural disasters, arable land reduction, unpredictable weather and rising temperatures, which may further aggravate the weak governance of the country. At present, the research on the fragile states or the study on the national vulnerabilities has become a basic core topic when the academic circles discuss the issue of world development. To some extent, the effectiveness of the international community in dealing with the issue of fragile states shows the progress and limits of global governance.

\section{Model Application in Yemen}

We choose Yemen as our target of concern. Yemen is one of the world's most underdeveloped countries with a hot and humid climate, extremely scarce water resources, unstable political situation and backward economic development.

\subsection{Data Sources}

We need to collect twenty one data sets of metrics. Among these indicators, the data on the level of free citizens $\left(P A_{31}\right)$ and democratic level $\left(P A_{21}\right)$ are far from enough to meet our needs, so these two indicators can not be quantified. The main source of data is the World Bank. [1]

\subsection{Vulnerability Index and Climate Impact}

\subsection{Application of Gray Correlation Method}

Due to the complicated and indefinite correlation between the second class and first class indicators, here we use Gray relational analysis (GRA) to make analysis, GRA steps are as follows.

Normalization based on classification of indicators. Normalization based on classification of indicators. We know that except "less is better" indicators such as unemployment rate, refugees, crime index and religious conflicts, others are "higher and better" indicators. So, we can adopt the method of maximum difference normalization for the "higher is better" indicators. 


$$
Z_{i j}=\frac{S_{i j}-S_{i \min }}{S_{i \max }-S_{i \min }}
$$

And we can have the method of minimum difference normalization for" lower is better" indicators.

$$
Z_{i j}=\frac{S_{i \max }-S_{i j}}{S_{i \max }-S_{i \min }}
$$

where $S_{i j}$ is the $j$ th country observation on the ith indicator.

Choose the reference series. The reference series consists of the best values for each indicator. We use the average of indicators from the reference cities we select, South Sudan, Somalia etc. The reference series $z_{0}=\left\{z_{01}^{1}, z_{02}^{1}, \cdots, z_{31}^{3}, z_{32}^{3}\right\}$.

Compute grey rational coefficient $\gamma_{i j}$ with respect to the jth second class indicator of the firstclass indicator. The equation is

$$
\gamma_{i j}=\frac{\Delta_{\min }+\tau \Delta_{\max }}{\Delta_{i j}+\tau \Delta_{\max }}
$$

where $\Delta_{i j}=z_{i j}-z_{0 j}, \Delta_{\max }=\max _{i} \max _{j} \Delta_{i j}, \Delta_{\min }=\min _{i} \min _{j} \Delta_{i j}$, and resolution ratio $\tau$ is set 0.5 to optimally improve the significance of the difference of the difference among rational coefficients.

Calculate the rational grade of each first class indicator respectively by taking the average of its rational coefficient.

$$
\delta_{i}=\frac{1}{n} \sum_{i=1}^{n} \gamma_{i j}, j=1, \cdots, n
$$

where $\mathrm{n}$ is the number of second class indicators of the ith first class indicator.

The vulnerability grade demonstrates the connection between the country we research and those that are more vulnerable. The greater the degree of correlation of indicators, the higher the vulnerability of the country.

After the processing of data, we calculate the vulnerability grade of each first class indicator of the country. We show the GRA results as follows.

Table 1. Vulnerability Grade of Each Metric in Yemen

\begin{tabular}{c|c|c|c|c|c|c|c|c}
\hline$S A_{1}$ & $S A_{2}$ & $S A_{3}$ & $E A_{1}$ & $E A_{2}$ & $E A_{3}$ & $P A_{1}$ & $P A_{2}$ & $P A_{3}$ \\
\hline 0.9524 & 0.9047 & 0.8863 & 0.8147 & 0.9231 & 0.8011 & 0.8554 & 0.8998 & 0.8762 \\
\hline
\end{tabular}

After calculating the average of each metric, we get $S A(0.9145), E A(0.8463), P A(0.8768)$, whose weighted average with weight $(0.4,0.3,0.3)$ is 0.8827 .

In order to measure the degree of vulnerability of a country, we use the long-term fragile states as reference standards and define the following four vulnerabilities. [2]

Fragile: with value of vulnerability index from 0.76 to 1 .

Vulnerable: with value of vulnerability index between from 0.51 to 0.75 .

Stable: with value of vulnerability index between from 0.26 to 0.5 .

Sustainable: with value of vulnerability index between from 0 to 0.25 . 


\subsection{Impact of Climate Change on Second Class Indicators}

Using regression analysis, we analyze the effects of precipitation (x) and temperature (y) [3] on the four second class indicators of per capita water resources, crime index[4], per capita GDP, and infrastructure. And we fit the corresponding functional relationships and make functional images.

Per capita water resources.

$$
f(x, y)=-22.81+6.585 x+0.4735 y
$$

From the function, we can conclude that the temperature has less influence on the per capita water resources, and as the precipitation increases, the per capita water resource increases accordingly.
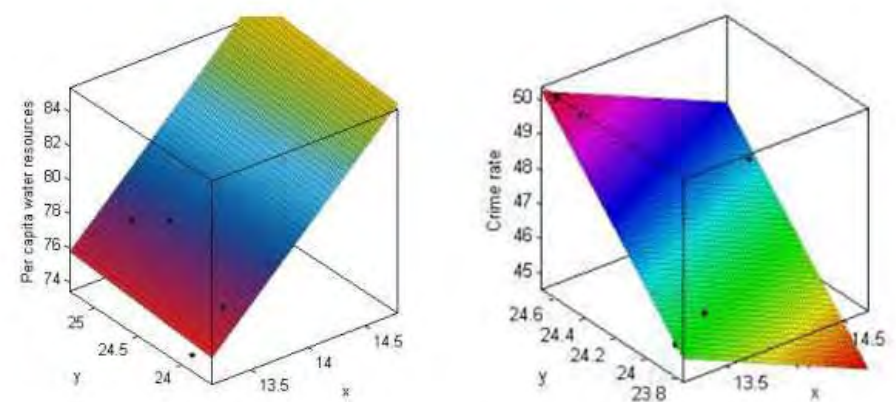

Figure 1. Per capita water resources and crime index images.

Crime index.

$$
f(x, y)=-63.49-1.491 x+5.401 y
$$

Combined with images and functions, we conclude that the higher the temperature, the less precipitation, the higher the crime index. [4]

GDP per capita.

$$
f(x, y)=4.385 \times 10^{5}-3.278 \times 10^{4} x-1.758 \times 10^{4} y+56.79 x^{2}+1288 x y
$$

By analyzing the images and functions, we conclude that the higher the precipitation is, the higher the per capita GDP is over a certain temperature range.

Infrastructure.

$$
f(x, y)=106.3+14.91 x-2.927 y
$$

The impact of temperature on infrastructure is small, and as the amount of precipitation increases, the infrastructure tends to improve.

\subsection{Impact of Climate Change on the Vulnerability Index}

The direct impact of climate change on the secondary indicators has indirectly led to changes in the country's vulnerability.

Yemen has high temperatures throughout the year and very few rainfall, resulting in per capita water resources, per capita GDP, and infrastructure at a relatively low level all year long, while the crime index is at a relatively high level. In turn, the country's vulnerability is greatly enhanced.

If Yemen is not affected by these adverse weather conditions, that is, precipitation and temperature are in the right range, it will raise per capita water resources, per capita GDP, and infrastructure levels, 
and reduce the crime index, thus lowering the vulnerability index and improving the stability of the country.

\subsection{Human Intervention}

Most of the Yemen tropical desert climate, in the summer there are sandstorms common in the desert, precipitation is scarce, prone to drought. In order to reduce the damage caused to countries by natural disasters caused by climate change, we propose the following interventions and analyze the impact they have on national vulnerabilities.

Emphasis on water conservancy projects and strengthen the construction of underground tanks. This measure is conducive to alleviating the risks posed by drought, ensuring a certain level of per capita water resources, alleviating population pressure and alleviating the country's vulnerability.

Promote advanced irrigation technology, the establishment of underground pipe drip irrigation systems. The measures will help improve the efficiency of water resources utilization, reduce water consumption to some extent, and ease the pressure of water resources. At the same time, efficient irrigation increases crop yields and increases per capita GDP. National vulnerability reduced.

Vigorously build farm shelterbelts network to protect the existing vegetation. This measure is conducive to alleviating the desertification of land, maintaining soil and water and reducing the losses caused by sandstorms to life and production, thereby increasing per capita GDP and reducing the country’s vulnerability.

\section{Evaluation of the Model}

\subsection{Strengths}

Based on the literature and the actual situation, we describe in detail the applicability of the National Vulnerability Assessment System and its three components: SA, EA and PA.

Based on the two factors of temperature and precipitation, we fully analyze the impact of climate change on national vulnerability.

We use gray correlation and regression analysis to analyze and predict the data, so our model is convincing.

We make full use of tables and charts to make the analysis process and results clear.

\subsection{Weaknesses}

Data deviation: The data we collect comes from multiple websites and differences in statistical standards can lead to bias conclusions. Moreover, the lack of data for more indicators can lead to errors in the assessment system.

Subjectivity: There are many subjective approaches in the model, and some indicators are based on our own experience and intuition. Some may not be credible.

In the forecasting process, we believe that the second class indicators that are not affected by the weather remain stable, which is in fact deviating from the actual indicators.

\section{Conclusion}

In this paper, we consider a country's vulnerability indicator. In the case of Yemen, we analyzed in detail how climate change indirectly affects national vulnerabilities by affecting Per capita water resources, Crime index, GDP per capita and infrastructure. First, we set up National Vulnerability Assessment System by referring to FSI. We divide NVAS into three components, namely social aspects, economic aspects, political aspects. And developed the World Bank data, using the gray correlation analysis to get the NV index. Followed by regression analysis to find the precipitation and temperature and the above four indicators of the regression equation. As a result, the impact of climate change on the vulnerability of Yemen has been obtained and relevant human interventions have been proposed to mitigate the impact. Finally, we analyze and summarize the advantages and disadvantages of the model. 


\section{References}

[1]. Information on: https://data.worldbank.org.

[2]. Tianxu Liu, Tao $\mathrm{Wu}$, Evaluation criteria for vulnerable countries [J]. Lead the science forum, 2016(13):17-26.

[3]. Information on: http://sdwebx.worldbank.org/climateportal/.

[4]. Robert Agnew, Dire forecast: A theoretical model of the impact of climate change on crime, Theoretical Criminology Vol 16,21-42. 